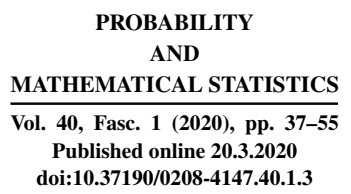

\title{
A NOTE ON EXISTENCE OF GLOBAL SOLUTIONS AND INVARIANT MEASURES FOR JUMP SDES WITH LOCALLY ONE-SIDED LIPSCHITZ DRIFT
}

\author{
BY
}

\author{
MATEUSZ B. MA J KA (BONN)
}

\begin{abstract}
We extend some methods developed by Albeverio, Brzeźniak and $\mathrm{Wu}$ and we show how to apply them in order to prove existence of global strong solutions of stochastic differential equations with jumps, under a local one-sided Lipschitz condition on the drift (also known as a monotonicity condition) and a local Lipschitz condition on the diffusion and jump coefficients, while an additional global one-sided linear growth assumption is satisfied. Then we use these methods to prove existence of invariant measures for a broad class of such equations.
\end{abstract}

2020 Mathematics Subject Classification: Primary 60H10; Secondary 60G51.

Key words and phrases: stochastic differential equations, invariant measures, jump processes.

\section{EXISTENCE OF GLOBAL SOLUTIONS UNDER LOCAL LIPSCHITZ CONDITIONS}

Consider a stochastic differential equation in $\mathbb{R}^{d}$ of the form

$$
d X_{t}=b\left(X_{t}\right) d t+\sigma\left(X_{t}\right) d W_{t}+\int_{U} g\left(X_{t-}, u\right) \tilde{N}(d t, d u) .
$$

Here $b: \mathbb{R}^{d} \rightarrow \mathbb{R}^{d}, \sigma: \mathbb{R}^{d} \rightarrow \mathbb{R}^{d \times d}, g: \mathbb{R}^{d} \times U \rightarrow \mathbb{R}^{d},\left(W_{t}\right)_{t \geqslant 0}$ is a $d$-dimensional Wiener process, $(U, \mathcal{U}, \nu)$ is a $\sigma$-finite measure space and $N(d t, d u)$ is a Poisson random measure on $\mathbb{R}^{+} \times U$ with intensity measure $d t \nu(d u)$, while $\widetilde{N}(d t, d u)=$ $N(d t, d u)-d t \nu(d u)$ is the compensated Poisson random measure. We denote by $\left(X_{t}(x)\right)_{t \geqslant 0}$ a solution to 1.1 with initial condition $x \in \mathbb{R}^{d}$. The main result of the present paper is Theorem 2.1. where we prove existence of invariant measures for a certain class of such equations.

However, we first discuss the existence of strong solutions to (1.1), in the context of the paper [1] by Albeverio, Brzeźniak and $\mathrm{Wu}$. We will prove the following result, where $\|\cdot\|_{\text {HS }}$ denotes the Hilbert-Schmidt norm of a matrix. 
THEOREM 1.1. Assume that the coefficients in (1.1) satisfy the following local one-sided Lipschitz condition: for every $R>0$ there exists $C_{R}>0$ such that for any $x, y \in \mathbb{R}^{d}$ with $|x|,|y| \leqslant R$ we have

$$
\begin{array}{r}
\langle b(x)-b(y), x-y\rangle+\|\sigma(x)-\sigma(y)\|_{\mathrm{HS}}^{2}+\int_{U}|g(x, u)-g(y, u)|^{2} \nu(d u) \\
\leqslant C_{R}|x-y|^{2}
\end{array}
$$

Moreover, assume a global one-sided linear growth condition: there exists $C>0$ such that for any $x \in \mathbb{R}^{d}$ we have

$$
\langle b(x), x\rangle+\|\sigma(x)\|_{\mathrm{HS}}^{2}+\int_{U}|g(x, u)|^{2} \nu(d u) \leqslant C\left(1+|x|^{2}\right) .
$$

If in addition $b: \mathbb{R}^{d} \rightarrow \mathbb{R}^{d}$ is continuous, then there exists a unique global strong solution to 1.1 .

The one-sided Lipschitz condition (1.2) above is sometimes called a monotonicity condition (see e.g. [7] or [11]), a weak monotonicity condition ([12] or [16]) or a dissipativity condition ([13], [17] or [20]), although the term "dissipativity" is often restricted to the case where (1.2) is satisfied with $C_{R}<0$. We call (1.2) one-sided Lipschitz condition regardless of the sign of the constant, and use the term "dissipativity" only if the constant is negative. Note that the above theorem is a generalization of the following classical result.

THEOREM 1.2. Assume that the coefficients in (1.1) satisfy a global Lipschitz condition: there exists $C>0$ such that for any $x, y \in \mathbb{R}^{d}$ we have

$$
|b(x)-b(y)|^{2}+\|\sigma(x)-\sigma(y)\|_{\mathrm{HS}}^{2}+\int_{U}|g(x, u)-g(y, u)|^{2} \nu(d u) \leqslant C|x-y|^{2} .
$$

Moreover, assume a global linear growth condition: there exists $L>0$ such that for any $x \in \mathbb{R}^{d}$ we have

$$
|b(x)|^{2}+\|\sigma(x)\|_{\mathrm{HS}}^{2}+\int_{U}|g(x, u)|^{2} \nu(d u) \leqslant L\left(1+|x|^{2}\right) .
$$

Then there exists a unique strong solution to (1.1.

Theorem 1.2 is very well-known and its proof can be found in many textbooks: see e.g. [9, Theorem IV-9.1] or [2, Theorem 6.2.3]. Note that we present it here as it appears in [9] and [2], but if (1.4) holds, then the linear growth condition on $b$ and $\sigma$ is redundant and $(1.5)$ can be replaced by a weaker integrability condition on $g$ (cf. the discussion in [1, Section 2], in particular Corollary 2.1 therein). On the other hand, Theorem 1.1 is not so popular and we had problems finding a suitable reference. We finally learned that Theorem 1.1 can be inferred from [7, Theorem 2], where a more general result is proved for equations driven by locally square integrable càdlàg martingales taking values in Hilbert spaces. 
Nevertheless, many authors use existence of solutions to equations like (1.1) under a one-sided Lipschitz condition for the drift (see e.g. [13], [17], [19], [20], to name only some recent papers) claiming that this result is well-known, without giving any proper reference. Books that cover this context include e.g. [4] and [18] which, admittedly, contain various interesting extensions of Theorem 1.2 , but not one where the Lipschitz condition is replaced with one-sided Lipschitz condition and the linear growth with one-sided linear growth. Moreover, Albeverio, Brzeźniak and $\mathrm{Wu}$ [1, Theorem 3.1] proved the following.

THEOREM 1.3. Assume that for any $R>0$ there exists $C_{R}>0$ such that for any $x, y \in \mathbb{R}^{d}$ with $|x|,|y| \leqslant R$ we have

$$
|b(x)-b(y)|^{2}+\|\sigma(x)-\sigma(y)\|_{\mathrm{HS}}^{2} \leqslant C_{R}|x-y|^{2},
$$

and there exists $L>0$ such that for any $x, y \in \mathbb{R}^{d}$ we have

$$
\int_{U}|g(x, u)-g(y, u)|^{2} \nu(d u) \leqslant L|x-y|^{2} .
$$

Finally, assume the global one-sided linear growth condition $(1.3)$ : there exists $C>0$ such that for any $x \in \mathbb{R}^{d}$ we have

$$
\langle b(x), x\rangle+\|\sigma(x)\|_{\mathrm{HS}}^{2}+\int_{U}|g(x, u)|^{2} \nu(d u) \leqslant C\left(1+|x|^{2}\right) .
$$

Then there exists a unique global strong solution to 1.1 .

It is clear that Theorem 1.3 is less general than Theorem 1.1 and thus it is also a special case of [7, Theorem 2]. Nevertheless, the proof in [1] is clearer and more direct than the one in [7]: the result in [7] is obtained by showing that (1.1) can be represented as an equation driven by certain Hilbert-space-valued martingales, whereas in [1] all the calculations are done directly for integrals with respect to Poisson random measures. The main idea in [1] is to modify the locally Lipschitz coefficients in such a way as to obtain globally Lipschitz functions that agree with the given coefficients on a ball of fixed radius. Then using Theorem 1.2 one can obtain a solution in every such ball and then "glue" such local solutions by using the global one-sided linear growth condition to obtain a global solution. It is important to mention that the authors of [1] also use their methods to prove existence of invariant measures for a broad class of equations of the form (1.1).

In view of all the above comments, we find it necessary to give a direct proof of Theorem 1.1. In the spirit of the proof in [1], we show how to extend the classical result (Theorem 1.2) step by step to obtain Theorem 1.1. Then we explain how to use the methods of [1] to obtain existence of invariant measures in our case (see Theorem 2.1). This is a new result with potential applications in the theory of SPDEs (see Example 2.2).

To prove both Theorems 1.1 and 2.1 we need the following auxiliary result regarding a possible modification of the coefficients in (1.1). 
LEMMA 1.1. Assume that the coefficients in 1.1. satisfy the local one-sided Lipschitz condition (1.2) and are locally bounded, i.e., for every $R>0$ there exists an $M_{R}>0$ such that for all $x \in \mathbb{R}^{d}$ with $|x| \leqslant R$ we have

$$
|b(x)|^{2}+\|\sigma(x)\|_{\mathrm{HS}}^{2}+\int_{U}|g(x, u)|^{2} \nu(d u) \leqslant M_{R} .
$$

Then for every $R>0$ there exist functions $b_{R}: \mathbb{R}^{d} \rightarrow \mathbb{R}^{d}, \sigma_{R}: \mathbb{R}^{d} \rightarrow \mathbb{R}^{d \times d}$ and $g_{R}: \mathbb{R}^{d} \times U \rightarrow \mathbb{R}^{d}$ such that for all $x \in \mathbb{R}^{d}$ with $|x| \leqslant R$ we have

$$
b_{R}(x)=b(x), \sigma_{R}(x)=\sigma(x) \text { and } g_{R}(x, u)=g(x, u) \text { for all } u \in \mathbb{R}^{d} .
$$

Moreover, $b_{R}, \sigma_{R}$ and $g_{R}$ satisfy a global one-sided Lipschitz condition: there exists a constant $C(R)>0$ such that for all $x, y \in \mathbb{R}^{d}$ we have

$$
\begin{aligned}
\left\langle b_{R}(x)-b_{R}(y), x-\right. & y\rangle+\left\|\sigma_{R}(x)-\sigma_{R}(y)\right\|_{\mathrm{HS}}^{2} \\
& +\int_{U}\left|g_{R}(x, u)-g_{R}(y, u)\right|^{2} \nu(d u) \leqslant C(R)|x-y|^{2}
\end{aligned}
$$

and they are globally bounded in the sense that there exists $M(R)>0$ such that for all $x \in \mathbb{R}^{d}$ we have

$$
\left|b_{R}(x)\right|^{2}+\left\|\sigma_{R}(x)\right\|_{\mathrm{HS}}^{2}+\int_{U}\left|g_{R}(x, u)\right|^{2} \nu(d u) \leqslant M(R) .
$$

Then, combining Theorem 1.2 and Lemma 1.1, we prove existence of solutions when the coefficients in (1.1) are bounded and satisfy a global one-sided Lipschitz condition.

THEOREM 1.4. Assume that $b$ is continuous and that the coefficients in (1.1) satisfy a global one-sided Lipschitz condition: there exists $K>0$ such that for all $x, y \in \mathbb{R}^{d}$ we have

$$
\begin{array}{r}
\langle b(x)-b(y), x-y\rangle+\|\sigma(x)-\sigma(y)\|_{\mathrm{HS}}^{2}+\int_{U}|g(x, u)-g(y, u)|^{2} \nu(d u) \\
\leqslant K|x-y|^{2} .
\end{array}
$$

Additionally, assume that the coefficients are globally bounded: there exists $M>0$ such that for all $x \in \mathbb{R}^{d}$ we have

$$
|b(x)|^{2}+\|\sigma(x)\|_{\mathrm{HS}}^{2}+\int_{U}|g(x, u)|^{2} \nu(d u) \leqslant M .
$$

Then there exists a unique strong solution to (1.1).

The proofs of Lemma 1.1 and Theorem 1.4 can be found in Section 3 . After proving the above results, we proceed with the proof of Theorem 1.1 as in [1, Proposition 2.9 and Theorem 3.1] (see also [7, p. 14] for a similar reasoning). More details can be found at the end of Section 3 . 


\section{EXISTENCE OF INVARIANT MEASURES}

The existence of an invariant measure for the solution of (1.1) is shown using the Krylov-Bogolyubov method (see e.g. [8, Theorem III-2.1] and the discussion in the introduction to [6]). For the existence of an invariant measure for a process $\left(X_{t}\right)_{t \geqslant 0}$ with a Feller semigroup $\left(p_{t}\right)_{t \geqslant 0}$ it is sufficient to show that for some $x \in \mathbb{R}^{d}$ the process $\left(X_{t}(x)\right)_{t \geqslant 0}$ is bounded in probability at infinity in the sense that for any $\varepsilon>0$ there exist $R, t>0$ such that for all $s \geqslant t$ we have

$$
\mathbb{P}\left(\left|X_{s}(x)\right|>R\right)<\varepsilon .
$$

Therefore if we show that there exist constants $M, K>0$ such that

$$
\mathbb{E}\left|X_{t}(x)\right|^{2} \leqslant|x|^{2} e^{-K t}+M / K
$$

for all $t \geqslant 0$, then 2.1 follows easily by the Chebyshev inequality and we obtain the existence of an invariant measure. Based on this idea, we can prove the following result.

THEOREM 2.1. Assume that the coefficients in (1.1) satisfy the local one-sided Lipschitz condition (1.2) and that there exist constants $K, M>0$ such that for all $x \in \mathbb{R}^{d}$ we have

$$
2\langle b(x), x\rangle+\|\sigma(x)\|_{\mathrm{HS}}^{2}+\int_{U}|g(x, u)|^{2} \nu(d u) \leqslant-K|x|^{2}+M .
$$

Assume also that there exists a constant $L>0$ such that for all $x \in \mathbb{R}^{d}$ we have

$$
\|\sigma(x)\|_{\mathrm{HS}}^{2}+\int_{U}|g(x, u)|^{2} \nu(d u) \leqslant L\left(1+|x|^{2}\right) .
$$

Finally, suppose that the drift coefficient $b$ in (1.1) is continuous. Then there exists an invariant measure for the solution of (1.1).

We can compare this result with [1, Theorem 4.5].

THEOREM 2.2. Assume that the coefficients $b$ and $\sigma$ in 11.1 satisfy the local Lipschitz condition (1.6) and that $g$ satisfies the global Lipschitz condition (1.7). Assume also the condition (2.3) as in Theorem 2.1. Then there exists an invariant measure for the solution of 1.1 .

REMARK 2.1. Observe that our additional condition (2.4) in Theorem 2.1] does not follow from 2.3) since $\langle b(x), x\rangle$ can be negative. Therefore it would seem that our result is not a straightforward generalization of [1, Theorem 4.5]. However, we believe that the condition (2.4) is also necessary for [1, Theorem 4.5], at least we have not been able to retrace the proof of Proposition 4.3 therein (which is crucial for the proof of Theorem 4.5) without this additional condition. Therefore we are convinced that 2.4 should be added to the list of assumptions of Theorem 4.5 
in [1] and that a result is indeed its strict generalization. This has been confirmed in a private communication with one of the authors of [1].

REMARK 2.2. Note that the constant 2 in (2.3) is not necessary, since obviously

$$
\begin{aligned}
2\langle b(x), x\rangle+\|\sigma(x)\|_{\mathrm{HS}}^{2} & +\int_{U}|g(x, u)|^{2} \nu(d u) \\
& \leqslant 2\langle b(x), x\rangle+2\|\sigma(x)\|_{\mathrm{HS}}^{2}+2 \int_{U}|g(x, u)|^{2} \nu(d u),
\end{aligned}
$$

and therefore if we replace (2.3) with

$$
\langle b(x), x\rangle+\|\sigma(x)\|_{\mathrm{HS}}^{2}+\int_{U}|g(x, u)|^{2} \nu(d u) \leqslant-K|x|^{2}+M,
$$

then (2.3) still holds with the right hand side multiplied by 2 and we can obtain 2.2 with $e^{-2 K t}$ instead of $e^{-K t}$ (see the proof of Theorem 2.1 and the calculations in the proof of Lemma 2.2 below). We have chosen to state this assumption in the form (2.3) to be consistent with [1], but note that we skip the constant 2 in all the other one-sided conditions involving the drift in our calculations.

For the proof of Theorem 2.1 we first need the following fact, which can be proved exactly as in [1, Propositions 4.1 and 4.2].

Lemma 2.1. The solution $\left(X_{t}\right)_{t \geqslant 0}$ to the equation (1.1) is a strong Markov process and thus it generates a Markov semigroup $\left(p_{t}\right)_{t \geqslant 0}$.

Now we need the following lemma, which is a generalization of [1, Proposition 4.3] (see Remark 2.1 about inclusion of the assumption (2.4)).

LEMMA 2.2. Under the assumptions (1.2), (1.3), (2.4) and if $b$ is continuous, the semigroup $\left(p_{t}\right)_{t \geqslant 0}$ associated with the solution $\left(X_{t}\right)_{t \geqslant 0}$ of $(1.1)$ is Feller.

Once we prove the above lemma, we can easily conclude the proof of Theorem 2.1, following [1, proof of Theorem 4.5], i.e., we just use the condition (2.3) to show (2.2) and then use the Krylov-Bogolyubov method above. More details can be found in Section 3 .

REMARK 2.3. Note that the Krylov-Bogolyubov method gives us a tool to prove existence of an invariant measure but does not provide any information about its uniqueness or the rate of convergence of distributions of the solution of (1.1) to equilibrium. However, if we replace (2.3) with a dissipativity at infinity condition, which is a natural (although much stronger) counterpart of (2.3), i.e., if we assume that there exist $K, R>0$ such that for all $x, y \in \mathbb{R}^{d}$ with $|x-y|>R$ we have

$$
\begin{array}{r}
\langle b(x)-b(y), x-y\rangle+\|\sigma(x)-\sigma(y)\|_{\mathrm{HS}}^{2}+\int_{U} \mid g(x, u)- \\
\left.\quad g(y, u)\right|^{2} \nu(d u) \\
\leqslant-K|x-y|^{2},
\end{array}
$$


then one can obtain uniqueness of the invariant measure. Namely, it has been proved in [14] by the coupling technique (see Corollary 2.7 therein) that for a certain class of SDEs of the form (1.1) satisfying (2.5) there exist constants $c>0$ and $C>1$ such that for any probability measures $\mu_{1}$ and $\mu_{2}$ on $\mathbb{R}^{d}$ and for any $t>0$ we have

$$
W_{1}\left(\mu_{1} p_{t}, \mu_{2} p_{t}\right) \leqslant C e^{-c t} W_{1}\left(\mu_{1}, \mu_{2}\right),
$$

where $W_{1}\left(\mu_{1}, \mu_{2}\right)$ is the standard $L^{1}$-Wasserstein distance between probability measures, $\left(p_{t}\right)_{t \geqslant 0}$ is the transition semigroup associated with the solution to 1.1 and $\mu p_{t}(d y)=\int \mu(d x) p_{t}(x, d y)$. Note that if 2.5$)$ holds for all $x, y \in \mathbb{R}^{d}$, then it is well-known that (2.6) holds (see e.g. [19, Theorem 1.1] and the references therein). It is easy to see that (2.6) implies existence of a unique invariant measure for $\left(p_{t}\right)_{t \geqslant 0}$ (see e.g. [10, Section 3]) and exponential rate of convergence to equilibrium in $W_{1}$. However, the condition (2.5) is not necessary for uniqueness of invariant measures, which has been obtained for certain classes of jump diffusions under other assumptions e.g. in [15, Corollary 5.4] or in [3, Section 5.1, in particular Remark 5.1]. See also [12] for uniqueness in the infinite-dimensional case without jumps under a weak dissipativity condition related to (2.5).

EXAMPLE 2.1. Fix $\alpha \in(0,1)$ and suppose that the drift in $\sqrt{1.1}$ is given by $b(x):=-x|x|^{-\alpha} \mathbf{1}_{\{x \neq 0\}}$. Equations of this type are considered in [18, Example 171]. It is easy to check that the function $b$ defined above is not locally Lipschitz, since it does not satisfy the Lipschitz condition in any neighbourhood of zero. However, we can show that it satisfies the one-sided Lipschitz condition globally with constant zero. Indeed, following the calculations in [18, Example 171], for any nonzero $x, y \in \mathbb{R}^{d}$ we have

$$
\begin{aligned}
\left\langle x-y,-x|x|^{-\alpha}+y|y|^{-\alpha}\right\rangle & =-|x|^{2-\alpha}+\left\langle y, x|x|^{-\alpha}\right\rangle+\left\langle x, y|y|^{-\alpha}\right\rangle-|y|^{2-\alpha} \\
& \leqslant-|x|^{2-\alpha}-|y|^{2-\alpha}+|y||x|^{1-\alpha}+|x||y|^{1-\alpha} \\
& =(|x|-|y|)\left(|y|^{1-\alpha}-|x|^{1-\alpha}\right) \leqslant 0
\end{aligned}
$$

where the last inequality holds since $1-\alpha \in(0,1)$. Thus, if we consider an equation of the form (1.1) with the drift $b$ and any locally Lipschitz coefficients $\sigma$ and $g$, the condition (1.2) is satisfied. Moreover, if $\sigma$ and $g$ satisfy the global linear growth condition (2.4) with some constant $L>0$, then by replacing the drift $b$ defined above with $b(x):=b(x)-K x$, where $K>L$, we obtain coefficients that satisfy (2.3). More generally, we can take $\widetilde{b}(x):=b(x)-\nabla U(x)$, where $U$ is a strongly convex function with convexity constant $K>L$. This way we obtain a class of examples of equations for which our Theorem 2.1 applies, but Theorem 4.5 in [1] does not, since the local Lipschitz assumption is not satisfied.

EXAMPLE 2.2. Our results may have applications in the study of stochastic evolution equations with Lévy noise on infinite-dimensional spaces, where the co- 
efficients are often non-Lipschitz (see e.g. [5] and the references therein). In particular, in [5] the authors consider SPDEs with drifts satisfying a local monotonicity condition and use their finite-dimensional approximations, which may lead to SDEs satisfying our condition (1.2) (cf. [5, condition (H2) and formula (4.4)].

\section{PROOFS}

In order to keep our presentation compact, we will only present the proof of Theorem 1.1 in a slightly less general setting than that presented in the first section. Namely, we will additionally assume that the diffusion coefficient $\sigma$ and the jump coefficient $g$ in (1.1) satisfy a local Lipschitz condition separately from the drift $b$, i.e., for every $R>0$ there exists $S_{R}>0$ such that for any $x, y \in \mathbb{R}^{d}$ with $|x|,|y| \leqslant R$ we have

$$
\|\sigma(x)-\sigma(y)\|_{\mathrm{HS}}^{2}+\int_{U}|g(x, u)-g(y, u)|^{2} \nu(d u) \leqslant S_{R}|x-y|^{2} .
$$

Obviously, 3.1 does not follow from (1.2), since $\langle b(x)-b(y), x-y\rangle$ may be negative. However, requiring (3.1) seems to be rather natural in many cases. It is possible to weaken this assumption and prove the exact statement of Theorem 1.1 using the methods of [11, Chapter II, Section 3] (see also [7, Section 3]), but this creates additional technical difficulties and thus we decided to omit this extension here, aiming at a clear and straightforward presentation.

Under the assumption (3.1), the coefficients of (1.1) automatically satisfy the local boundedness condition (1.8) required in Lemma 1.1 (recall that $b$ is assumed to be continuous and thus it is locally bounded anyway). It also means that from Lemma 1.1 we obtain coefficients $\sigma_{R}$ and $g_{R}$ that satisfy a separate global Lipschitz condition, i.e., 1.10 without the term involving $b_{R}$. Hence we can prove Theorem 1.4 under the additional assumption that there exists $S>0$ such that for all $x, y \in \mathbb{R}^{d}$ we have

$$
\|\sigma(x)-\sigma(y)\|_{\mathrm{HS}}^{2}+\int_{U}|g(x, u)-g(y, u)|^{2} \nu(d u) \leqslant S|x-y|^{2} .
$$

However, 3.1] is not needed for the proof of Theorem 2.1. where we also use Lemma 1.1, but we do not need to obtain truncated coefficients $\sigma_{R}$ and $g_{R}$ satisfying a separate global Lipschitz condition, and the local boundedness assumption is guaranteed by the separate linear growth condition (2.4) and the continuity of $b$. Thus the reasoning presented below gives a complete proof of the exact statement of our Theorem 2.1.

Proof of Lemma 1.1. For a related reasoning, see [7, proof of Lemma 4] or [18, Lemma 172]. Note that the method of truncating the coefficients of (1.1) which was used in [1, proof of Proposition 2.7] and which works in the case of Lipschitz 
coefficients, does not work for a one-sided Lipschitz drift and thus we need a different approach. For any $R>0$, we can consider a smooth, non-negative function $\eta_{R} \in \mathcal{C}_{c}^{\infty}\left(\mathbb{R}^{d}\right)$ such that

$$
\eta_{R}(x)= \begin{cases}1 & \text { if }|x| \leqslant R \\ 0 & \text { if }|x|>R+1\end{cases}
$$

and $\eta_{R}(x) \leqslant 1$ for all $x \in \mathbb{R}^{d}$. Then we can define

$$
b_{R}(x):=\eta_{R}(x) b(x), \sigma_{R}(x):=\eta_{R}(x) \sigma(x), \quad g_{R}(x, u):=\eta_{R}(x) g(x, u)
$$

for all $u \in U$. Then the condition (1.9) is obviously satisfied, and (1.11) immediately follows from (1.8). Therefore it remains to show that the functions $b_{R}, \sigma_{R}$ and $g_{R}$ satisfy the global one-sided Lipschitz condition (1.10). We have

$$
\begin{aligned}
& \left\langle b_{R}(x)-b_{R}(y), x-y\right\rangle+\left\|\sigma_{R}(x)-\sigma_{R}(y)\right\|_{\mathrm{HS}}^{2}+\int_{U}\left|g_{R}(x, u)-g_{R}(y, u)\right|^{2} \nu(d u) \\
& =\left\langle\eta_{R}(x) b(x)-\eta_{R}(y) b(y), x-y\right\rangle+\left\|\eta_{R}(x) \sigma(x)-\eta_{R}(y) \sigma(y)\right\|_{\mathrm{HS}}^{2} \\
& \quad+\int_{U}\left|\eta_{R}(x) g(x, u)-\eta_{R}(y) g(y, u)\right|^{2} \nu(d u) \\
& \leqslant \\
& \quad \eta_{R}(x)\langle b(x)-b(y), x-y\rangle+\left\langle\left(\eta_{R}(x)-\eta_{R}(y)\right) b(y), x-y\right\rangle \\
& \quad+\left|\eta_{R}(x)\right|^{2}\|\sigma(x)-\sigma(y)\|_{\mathrm{HS}}^{2}+\left\|\left(\eta_{R}(x)-\eta_{R}(y)\right) \sigma(y)\right\|_{\mathrm{HS}}^{2} \\
& \quad+\int_{U}\left|\eta_{R}(x)\right|^{2}|g(x, u)-g(y, u)|^{2} \nu(d u)+\int_{U}\left|\eta_{R}(x)-\eta_{R}(y)\right|^{2}|g(y, u)|^{2} \nu(d u) .
\end{aligned}
$$

Now assume that $\eta_{R}(y) \geqslant \eta_{R}(x)>0$ (the case when $\eta_{R}(x)=0$ is simpler and the case $\eta_{R}(y) \leqslant \eta_{R}(x)$ can be handled by interchanging $x$ and $y$ ). This implies that $|x|,|y| \leqslant R+1$ and thus we can use the local one-sided Lipschitz condition (1.2) with $R+1$ to get

$$
\begin{array}{r}
\langle b(x)-b(y), x-y\rangle+\|\sigma(x)-\sigma(y)\|_{\mathrm{HS}}^{2}+\int_{U}|g(x, u)-g(y, u)|^{2} \nu(d u) \\
\quad \leqslant C_{R+1}|x-y|^{2}
\end{array}
$$

with some constant $C_{R+1}$. Combining this with the fact that $\eta_{R} \leqslant 1$ (and thus $\eta_{R}^{2} \leqslant \eta_{R}$ ) allows us to bound the sum of the first, third and fifth terms on the right hand side of 3.4 by $C_{R+1}|x-y|^{2}$. Observe now that $\eta_{R}$ is Lipschitz (say with a constant $\left.C_{\operatorname{Lip}\left(\eta_{R}\right)}\right)$ and thus $\eta_{R}(x)-\eta_{R}(y) \leqslant C_{\operatorname{Lip}\left(\eta_{R}\right)}|x-y|$. Since $|y| \leqslant R+1$, we can use the local boundedness condition $(1.8)$ with some constant $M_{R+1}$. We first bound $|b(y)|$ by the square root of the left hand side of $[1.8)$ in order to get

$$
\left\langle\left(\eta_{R}(x)-\eta_{R}(y)\right) b(y), x-y\right\rangle \leqslant \sqrt{M_{R+1}} C_{\operatorname{Lip}\left(\eta_{R}\right)}|x-y|^{2} .
$$


Then we use (1.8) once again in order to bound the sum of the fourth and sixth terms on the right hand side of 3.4 by $M_{R+1} C_{\operatorname{Lip}\left(\eta_{R}\right)}^{2}|x-y|^{2}$. Combining these, we bound the right hand side of (3.4) by

$$
C_{R+1}|x-y|^{2}+\sqrt{M_{R+1}} C_{\operatorname{Lip}\left(\eta_{R}\right)}|x-y|^{2}+M_{R+1} C_{\operatorname{Lip}\left(\eta_{R}\right)}^{2}|x-y|^{2} .
$$

Therefore the global one-sided Lipschitz condition for $b_{R}, \sigma_{R}$ and $g_{R}$ is satisfied with the constant $C(R):=C_{R+1}+\sqrt{M_{R+1}} C_{\operatorname{Lip}\left(\eta_{R}\right)}+M_{R+1} C_{\operatorname{Lip}\left(\eta_{R}\right)}^{2}$, which finishes the proof.

LEMMA 3.1. Assume that the coefficients of the equation (1.1) with an initial condition $x \in \mathbb{R}^{d}$ satisfy the global one-sided linear growth condition $\sqrt{1.3}$ and that $\sigma$ and $g$ additionally satisfy the separate linear growth condition (2.4). Then there exist constants $\widetilde{C}, \widetilde{K}>0$ such that

$$
\mathbb{E} \sup _{s \leqslant t}\left|X_{s}\right|^{2} \leqslant \widetilde{K} e^{2 \widetilde{C} t}\left(1+|x|^{2}\right),
$$

where $\left(X_{t}\right)_{t \geqslant 0}=\left(X_{t}(x)\right)_{t \geqslant 0}$ is a solution to 1.1 with initial value $x \in \mathbb{R}^{d}$.

Proof. By the Itô formula, we have

$$
\begin{aligned}
\left|X_{t}\right|^{2}= & |x|^{2}+2 \int_{0}^{t}\left\langle X_{s}, b\left(X_{s}\right)\right\rangle d s+2 \int_{0}^{t}\left\langle X_{s}, \sigma\left(X_{s}\right) d W_{s}\right\rangle \\
& +\int_{0}^{t}\left\|\sigma\left(X_{s}\right)\right\|_{\mathrm{HS}}^{2} d s+2 \int_{0}^{t} \int_{U}\left\langle X_{s}, g\left(X_{s-}, u\right)\right\rangle \tilde{N}(d s, d u) \\
& +\int_{0}^{t} \int_{U}\left|g\left(X_{s-}, u\right)\right|^{2} N(d s, d u) .
\end{aligned}
$$

Now define $M_{t}:=\int_{0}^{t}\left\langle X_{s}, \sigma\left(X_{s}\right) d W_{s}\right\rangle+\int_{0}^{t} \int_{U}\left\langle X_{s}, g\left(X_{s-}, u\right)\right\rangle \tilde{N}(d s, d u)$, which is a local martingale. By the Burkholder-Davis-Gundy inequality, there exists a constant $C_{1}>0$ such that

$$
\begin{aligned}
& \mathbb{E} \sup _{s \leqslant t}\left|M_{s}\right| \leqslant C_{1} \mathbb{E}\left[\int_{0}^{t}\left|\sigma^{*}\left(X_{s}\right) X_{s}\right|^{2} d s+\int_{0}^{t} \int_{U}\left|\left\langle X_{s}, g\left(X_{s-}, u\right)\right\rangle\right|^{2} N(d s, d u)\right]^{1 / 2} \\
& \leqslant C_{1} \mathbb{E}\left[\left(\sup _{s \leqslant t}\left|X_{s}\right|^{2}\right)\left(\int_{0}^{t}\left\|\sigma^{*}\left(X_{s}\right)\right\|^{2} d s+\int_{0}^{t} \int_{U}\left|g\left(X_{s-}, u\right)\right|^{2} N(d s, d u)\right)\right]^{1 / 2} \\
& \leqslant C_{1}\left(\mathbb{E} \sup _{s \leqslant t}\left|X_{s}\right|^{2}\right)^{1 / 2}\left(\mathbb{E}\left[\int_{0}^{t}\left\|\sigma^{*}\left(X_{s}\right)\right\|^{2} d s+\int_{0}^{t} \int_{U}\left|g\left(X_{s-}, u\right)\right|^{2} N(d s, d u)\right]\right)^{1 / 2} \\
& \leqslant \frac{C_{1}}{2} a \mathbb{E} \sup _{s \leqslant t}\left|X_{s}\right|^{2}+\frac{C_{1}}{2 a} \mathbb{E}\left[\int_{0}^{t}\left\|\sigma^{*}\left(X_{s}\right)\right\|^{2} d s+\int_{0} \int_{U}\left|g\left(X_{s-}, u\right)\right|^{2} N(d s, d u)\right] \\
& \leqslant \frac{C_{1}}{2} a \mathbb{E} \sup _{s \leqslant t}\left|X_{s}\right|^{2}+\frac{C_{1}}{2 a} L \mathbb{E} \int_{0}^{t}\left(\left|X_{s}\right|^{2}+1\right) d s .
\end{aligned}
$$


Here $\|\cdot\|$ denotes the operator norm and $\sigma^{*}$ is the transpose of $\sigma$. In the third step we used the Hölder inequality in the form $\mathbb{E} A^{1 / 2} B^{1 / 2} \leqslant(\mathbb{E} A)^{1 / 2}(\mathbb{E} B)^{1 / 2}$, in the fourth step we used $(A B)^{1 / 2} \leqslant \frac{1}{2} a A+\frac{1}{2 a} B$ for any $a>0$, which can be chosen later, and in the fifth step we used the separate global linear growth condition (2.4) for $\sigma$ and $g$ along with the fact that $\|\cdot\| \leqslant\|\cdot\|_{\mathrm{HS}}$.

Now we can use (3.5) to get

$$
\begin{aligned}
\underset{s \leqslant t}{\mathbb{E} \sup _{s \leqslant}\left|X_{s}\right|^{2} \leqslant} & |x|^{2}+2 \mathbb{E} \sup _{s \leqslant t}\left|M_{s}\right|+2 \mathbb{E} \sup _{s \leqslant t} \int_{0}^{s}\left\langle X_{r}, b\left(X_{r}\right)\right\rangle d r \\
& +\mathbb{E} \sup _{s \leqslant t}\left[\int_{0}^{s}\left\|\sigma\left(X_{r}\right)\right\|_{\mathrm{HS}}^{2} d r+\int_{0}^{s} \int_{U}\left|g\left(X_{r-}, u\right)\right|^{2} N(d r, d u)\right] .
\end{aligned}
$$

Observe that obviously

$$
\left\langle X_{r}, b\left(X_{r}\right)\right\rangle \leqslant\left\langle X_{r}, b\left(X_{r}\right)\right\rangle+\left\|\sigma\left(X_{r}\right)\right\|_{\mathrm{HS}}^{2}+\int_{U}\left|g\left(X_{r-}, u\right)\right|^{2} \nu(d u)
$$

and thus from the global one-sided linear growth condition 1.3 we get

$$
\mathbb{E} \sup _{s \leqslant t} \int_{0}^{s}\left\langle X_{r}, b\left(X_{r}\right)\right\rangle d r \leqslant C \mathbb{E} \sup _{s \leqslant t} \int_{0}^{s}\left(\left|X_{r}\right|^{2}+1\right) d r \leqslant C \mathbb{E} \int_{0}^{t}\left(\left|X_{r}\right|^{2}+1\right) d r .
$$

On the other hand, using the separate linear growth condition 2.4 we get

$$
\begin{aligned}
\mathbb{E} \sup _{s \leqslant t}\left[\int_{0}^{s}\left\|\sigma\left(X_{r}\right)\right\|_{\mathrm{HS}}^{2}\right. & \left.d r+\int_{0}^{s} \int_{U}\left|g\left(X_{r-}, u\right)\right|^{2} N(d r, d u)\right] \\
& =\mathbb{E}\left[\int_{0}^{t}\left\|\sigma\left(X_{r}\right)\right\|_{\mathrm{HS}}^{2} d r+\int_{0}^{t} \int_{U}\left|g\left(X_{r-}, u\right)\right|^{2} N(d r, d u)\right] \\
& =\mathbb{E}\left[\int_{0}^{t}\left\|\sigma\left(X_{r}\right)\right\|_{\mathrm{HS}}^{2} d r+\int_{0}^{t} \int_{U}\left|g\left(X_{r-}, u\right)\right|^{2} \nu(d u) d r\right] \\
& \leqslant L \mathbb{E} \int_{0}^{t}\left(\left|X_{r}\right|^{2}+1\right) d r .
\end{aligned}
$$

Combining all the above estimates, we deduce from (3.6) that

$$
\mathbb{E} \sup _{s \leqslant t}\left|X_{s}\right|^{2} \leqslant|x|^{2}+C_{1} a \mathbb{E} \sup _{s \leqslant t}\left|X_{s}\right|^{2}+\left(\frac{C_{1}}{a} L+2 C+L\right) \mathbb{E} \int_{0}^{t}\left(\left|X_{r}\right|^{2}+1\right) d r
$$

Now, choosing $a=1 /\left(2 C_{1}\right)$ we obtain

$$
\mathbb{E} \sup _{s \leqslant t}\left|X_{s}\right|^{2} \leqslant 2|x|^{2}+2\left(2 C_{1}^{2} L+2 C+L\right) \mathbb{E} \int_{0}^{t} \sup _{w \leqslant r}\left(\left|X_{w}\right|^{2}+1\right) d r .
$$


Hence, using the Gronwall inequality for $\mathbb{E} \sup _{s \leqslant t}\left|X_{s}\right|^{2}+1$ we get

$$
\mathbb{E} \sup _{s \leqslant t}\left|X_{s}\right|^{2}+1 \leqslant 2\left(|x|^{2}+1\right) \exp \left(2\left(2 C_{1}^{2} L+2 C+L\right) t\right),
$$

which finishes the proof.

Proof of Theorem 1.4. Let $j \in \mathcal{C}_{c}^{\infty}\left(\mathbb{R}^{d}\right)$ have support contained in $B(0,1)$ and $\int_{\mathbb{R}^{d}} j(z) d z=1$. Then, for any $k \geqslant 1$, define

$$
b^{k}(x):=\int_{\mathbb{R}^{d}} b\left(x-\frac{z}{k}\right) j(z) d z .
$$

Now we can consider the sequence of equations

$$
d X_{t}^{k}=b^{k}\left(X_{t}^{k}\right) d t+\sigma\left(X_{t}^{k}\right) d W_{t}+\int_{U} g\left(X_{t-}^{k}, u\right) \tilde{N}(d t, d u) .
$$

Note that we have only replaced the drift coefficient $b$ with $b^{k}$, while $\sigma$ and $g$ remain unchanged. This is because we have decided to prove Theorem 1.1 with the additional assumption of separate local Lipschitz condition (3.1) for $\sigma$ and $g$. Thanks to this, we can work under the additional assumption that $\sigma$ and $g$ are globally Lipschitz, i.e., they satisfy (3.2) (see the discussion at the beginning of this section). Now observe that the function $b^{k}$ defined above is also globally Lipschitz. Indeed, for any $x, y \in \mathbb{R}^{d}$ we have

$$
\begin{aligned}
& \left|b^{k}(x)-b^{k}(y)\right|=\left|\int_{\mathbb{R}^{d}} b\left(x-\frac{z}{k}\right) j(z) d z-\int_{\mathbb{R}^{d}} b\left(y-\frac{z}{k}\right) j(z) d z\right| \\
& =\left|k^{d} \int_{B(x, 1 / k)} b(w) j(k(x-w)) d w-k^{d} \int_{B(y, 1 / k)} b(w) j(k(y-w)) d w\right| \\
& \leqslant k^{d} \int_{B(x, 1 / k) \cup B(y, 1 / k)}|b(w)||j(k(x-w))-j(k(y-w))| d w \\
& \leqslant 2 C_{d} k \sqrt{M}|x-y| \sup _{w \in \mathbb{R}^{d}}|\nabla j(w)|,
\end{aligned}
$$

where in the second step we use our assumption about the support of $j$ and in the last step we use the fact that $b$ is bounded by $\sqrt{M}$ (cf. $(1.13)$ ) and $j$ is Lipschitz with the Lipschitz constant given by the supremum of the norm of its gradient. Moreover, note that in the last step we bounded the volume of $B(x, 1 / k) \cup B(y, 1 / k)$ by $2 C_{d} / k^{d}$ for any $x, y \in \mathbb{R}^{d}$, where $C_{d}=\pi^{d / 2} / \Gamma(d / 2+1)$. Having proved that $b^{k}$ is globally Lipschitz, we can use Theorem 1.2 to ensure existence of a unique strong solution $\left(X_{t}^{k}\right)_{t \geqslant 0}$ to 3.8 .

We will prove now that the sequence $\left\{\left(X_{t}^{k}\right)_{t \geqslant 0}\right\}_{k=1}^{\infty}$ of solutions has a limit (in the sense of almost sure convergence, uniform on bounded time intervals) and that this limit is in fact a solution to (1.1). To this end, we will make use of the calculations from the proof of Lemma 3.1 . 
Observe that for any $k, l \geqslant 1$, if we use the Itô formula to calculate $\left|X_{t}^{k}-X_{t}^{l}\right|^{2}$, we will obtain exactly the formula 3.5 with $X_{t}$ replaced by $X_{t}^{k}-X_{t}^{l}$ and $b\left(X_{s}\right)$ replaced by $b^{k}\left(X_{s}^{k}\right)-b^{l}\left(X_{s}^{l}\right)$. Furthermore, we can make the term $|x|^{2}$ vanish (we can assume that all the solutions $\left(X_{t}^{k}\right)_{t \geqslant 0}$ have the same initial condition). Now we can proceed exactly as in the proof of Lemma 3.1, this time using the separate global Lipschitz condition (3.2) for $\sigma$ and $g$ where we used the separate linear growth condition (2.4) before, in order to get

$$
\begin{aligned}
\mathbb{E} \sup _{s \leqslant t}\left|X_{s}^{k}-X_{s}^{l}\right|^{2} \leqslant & C_{1} a \mathbb{E} \sup _{s \leqslant t}\left|X_{s}^{k}-X_{s}^{l}\right|^{2} \\
& +\left(\frac{C_{1}}{a} S+S\right) \mathbb{E} \int_{0}^{t}\left|X_{r}^{k}-X_{r}^{l}\right|^{2} d r \\
& +2 \mathbb{E} \sup _{s \leqslant t} \int_{0}^{s}\left\langle X_{r}^{k}-X_{r}^{l}, b^{k}\left(X_{r}^{k}\right)-b^{l}\left(X_{r}^{l}\right)\right\rangle d r .
\end{aligned}
$$

Thus the only term with which we have to deal in a different way compared to the proof of Lemma 3.1 is the last one. We have

$$
\begin{aligned}
& \mathbb{E} \sup _{s \leqslant t} \int_{0}^{s}\left\langle X_{r}^{k}-X_{r}^{l}, b^{k}\left(X_{r}^{k}\right)-b^{l}\left(X_{r}^{l}\right)\right\rangle d r \\
& =\mathbb{E} \sup _{s \leqslant t} \int_{0}^{s}\left\langle X_{r}^{k}-X_{r}^{l}, \int_{\mathbb{R}^{d}} b\left(X_{r}^{k}-\frac{z}{k}\right) j(z) d z-\int_{\mathbb{R}^{d}} b\left(X_{r}^{l}-\frac{z}{l}\right) j(z) d z\right\rangle d r \\
& =\mathbb{E} \sup _{s \leqslant t} \int_{0}^{s}\left\{\int_{\mathbb{R}^{d}}\left\langle\left(X_{r}^{k}-\frac{z}{k}\right)-\left(X_{r}^{l}-\frac{z}{l}\right), b\left(X_{r}^{k}-\frac{z}{k}\right)-b\left(X_{r}^{l}-\frac{z}{l}\right)\right\rangle j(z) d z\right. \\
& \left.\quad+\int_{\mathbb{R}^{d}}\left\langle\frac{z}{k}-\frac{z}{l}, b\left(X_{r}^{k}-\frac{z}{k}\right)-b\left(X_{r}^{l}-\frac{z}{l}\right)\right\rangle j(z) d z\right\} d r \\
& =: \mathbb{E} \sup _{s \leqslant t} \int_{0}^{s}\left(I_{r}^{1}+I_{r}^{2}\right) d r .
\end{aligned}
$$

Now observe that since $b$ is bounded by $\sqrt{M}$ (see 1.13 ), we have

$$
I_{r}^{2} \leqslant 2 \sqrt{M} \int_{\mathbb{R}^{d}}\left|\frac{z}{k}-\frac{z}{l}\right| j(z) d z=2 \sqrt{M}\left|\frac{1}{k}-\frac{1}{l}\right| \int_{\mathbb{R}^{d}}|z| j(z) d z .
$$

We denote $C^{1}(j):=\int_{\mathbb{R}^{d}}|z| j(z) d z$. As for $I_{r}^{1}$, we can use the one-sided Lipschitz condition 1.12 for $b$ similarly to the use of the one-sided linear growth in (3.7) to get

$$
\begin{aligned}
I_{r}^{1} & \leqslant K \int_{\mathbb{R}^{d}}\left|\left(X_{r}^{k}-\frac{z}{k}\right)-\left(X_{r}^{l}-\frac{z}{l}\right)\right|^{2} j(z) d z \\
& =K \int_{\mathbb{R}^{d}}\left|\left(X_{r}^{k}-X_{r}^{l}\right)-\left(\frac{1}{k}-\frac{1}{l}\right) z\right|^{2} j(z) d z \\
& \leqslant 2 K \int_{\mathbb{R}^{d}}\left|X_{r}^{k}-X_{r}^{l}\right|^{2} j(z) d z+2 K \int_{\mathbb{R}^{d}}\left|\frac{1}{k}-\frac{1}{l}\right|^{2}|z|^{2} j(z) d z .
\end{aligned}
$$


Recall that $\int_{\mathbb{R}^{d}} j(z) d z=1$ and denote $C^{2}(j):=\int_{\mathbb{R}^{d}}|z|^{2} j(z) d z$. Combining the above estimates, we have

$$
\begin{aligned}
\mathbb{E} \sup _{s \leqslant t} \int_{0}^{s}\left(I_{r}^{1}+I_{r}^{2}\right) d r \leqslant & 2 K \mathbb{E} \int_{0}^{t}\left|X_{r}^{k}-X_{r}^{l}\right|^{2} d r+2 t K C^{2}(j)\left|\frac{1}{k}-\frac{1}{l}\right|^{2} \\
& +2 t \sqrt{M}\left|\frac{1}{k}-\frac{1}{l}\right| C^{1}(j) \\
\leqslant & 2 K \mathbb{E} \int_{0}^{t} \sup _{w \leqslant r}\left|X_{w}^{k}-X_{w}^{l}\right|^{2} d r+\widehat{C} t\left|\frac{1}{k}-\frac{1}{l}\right|
\end{aligned}
$$

where the last inequality holds with a constant $\widehat{C}:=2 K C^{2}(j)+2 \sqrt{M} C^{1}(j)$ for $k$ and $l$ large enough that $\left|\frac{1}{k}-\frac{1}{l}\right|<1$. Now we come back to 3.9 and, taking $a=1 /\left(2 C_{1}\right)$, similarly to the proof of Lemma 3.1 we get

$$
\begin{aligned}
\mathbb{E} \sup _{s \leqslant t}\left|X_{s}^{k}-X_{s}^{l}\right|^{2} \leqslant & 2\left(2 C_{1}^{2} S+S\right) \mathbb{E} \int_{0}^{t} \sup _{w \leqslant r}\left|X_{w}^{k}-X_{w}^{l}\right|^{2} d r \\
& +8 K \mathbb{E} \int_{0}^{t} \sup _{w \leqslant r}\left|X_{w}^{k}-X_{w}^{l}\right|^{2} d r+4 \widehat{C} t\left|\frac{1}{k}-\frac{1}{l}\right| .
\end{aligned}
$$

The Gronwall inequality implies

$$
\mathbb{E} \sup _{s \leqslant t}\left|X_{s}^{k}-X_{s}^{l}\right|^{2} \leqslant 4 \widehat{C} t\left|\frac{1}{k}-\frac{1}{l}\right| \exp \left\{\left(4 C_{1}^{2} S+2 S+8 K\right) t\right\} .
$$

From this we can infer that there exists a process $\left(X_{t}\right)_{t \geqslant 0}$ such that

$$
\mathbb{E} \sup _{s \leqslant t}\left|X_{s}-X_{s}^{k}\right|^{2} \rightarrow 0 \quad \text { as } k \rightarrow \infty .
$$

It remains to show that $\left(X_{t}\right)_{t \geqslant 0}$ is indeed a solution to 1.1 . Observe that, by choosing a subsequence, we have $X_{t}^{k} \rightarrow X_{t}$ almost surely as $k \rightarrow \infty$, and thus, since $b$ is assumed to be continuous, we get

$$
b\left(X_{t}^{k}-\frac{z}{k}\right) \rightarrow b\left(X_{t}\right) \quad \text { almost surely as } k \rightarrow \infty .
$$

But $b$ is bounded by $\sqrt{M}$ and

$$
\int_{0}^{t} \int_{\mathbb{R}^{d}} b\left(X_{s}^{k}-\frac{z}{k}\right) j(z) d z d s \leqslant \sqrt{M} \int_{0}^{t} \int_{\mathbb{R}^{d}} j(z) d z d s<\infty .
$$

Therefore

$$
\int_{0}^{t} \int_{\mathbb{R}^{d}} b\left(X_{s}^{k}-\frac{z}{k}\right) j(z) d z d s \rightarrow \int_{0}^{t} \int_{\mathbb{R}^{d}} b\left(X_{s}\right) j(z) d z d s=\int_{0}^{t} b\left(X_{s}\right) d s
$$

as $k \rightarrow \infty$ a.s. Moreover, using the Itô isometry and (3.10), we can easily prove that $\int_{0}^{t} \sigma\left(X_{s}^{k}\right) d W_{s} \rightarrow \int_{0}^{t} \sigma\left(X_{s}\right) d W_{s}$ almost surely (by choosing a subsequence) and similarly $\int_{0}^{t} \int_{U} g\left(X_{s-}^{k}, u\right) \tilde{N}(d s, d u) \rightarrow \int_{0}^{t} \int_{U} g\left(X_{s-}, u\right) \tilde{N}(d s, d u)$ as $k \rightarrow \infty$, which finishes the proof. 
Proof of Lemma 2.2. First observe that under our assumptions, we can use Lemma 3.1 to get $\mathbb{E} \sup _{s \leqslant t}\left|X_{s}\right|^{2} \leqslant K_{1}\left(1+|x|^{2}\right) e^{K_{2} t}$ for any $t>0$ and some constants $K_{1}, K_{2}>0$, where $\left(X_{t}\right)_{t \geqslant 0}=\left(X_{t}(x)\right)_{t \geqslant 0}$ is a solution to 1.1 with initial condition $x \in \mathbb{R}^{d}$. Hence, by the Chebyshev inequality, for any $t>0$ and any $\varepsilon>0$ we can find $R>0$ large enough so that for any $x \in \mathbb{R}^{d}$ with $|x| \leqslant R$ we have

$$
\mathbb{P}\left(\sup _{s \leqslant t}\left|X_{s}(x)\right| \geqslant R\right) \leqslant \varepsilon / 2 .
$$

Now without loss of generality assume that $t \leqslant 1$ and fix $\varepsilon, R>0$ as above. We can consider a solution $\left(X_{t}^{R}\right)_{t \geqslant 0}$ to 1.1 with the coefficients replaced by the truncated coefficients $b_{R}, \sigma_{R}$ and $g_{R}$ obtained from Lemma 1.1 (note that the local boundedness assumption $(1.8)$ in Lemma 1.1 is satisfied due to the continuity of $b$ and the separate linear growth condition 2.4 for $\sigma$ and $g$, cf. the discussion at the beginning of this section). Then $b_{R}, \sigma_{R}$ and $g_{R}$ satisfy the global one-sided Lipschitz condition 1.10 with some constant $C(R)>0$. This in particular implies that the solution to the equation

$$
d X_{t}^{R}=b_{R}\left(X_{t}^{R}\right) d t+\sigma_{R}\left(X_{t}^{R}\right) d W_{t}+\int_{U} g_{R}\left(X_{t-}^{R}, u\right) \tilde{N}(d t, d u)
$$

is unique (Theorem 1.4). Hence, taking into account the fact that the coefficients $b_{R}, \sigma_{R}$ and $g_{R}$ agree on the ball of radius $R$ with their non-truncated counterparts (cf. 3.3 ), we see that $X_{s}=X_{s}^{R}$ for $s \leqslant \tau_{R}$ with $\tau_{R}$ defined by

$$
\tau_{R}:=\inf \left\{t>0:\left|X_{t}^{R}\right| \geqslant R\right\} .
$$

Thus for any $x, y \in \mathbb{R}^{d}$ with $|x|,|y| \leqslant R$ and for any $\delta>0$ we have

$$
\begin{aligned}
\mathbb{P}\left(\mid X_{1}(x)-\right. & \left.X_{1}(y) \mid>\delta\right) \\
= & \mathbb{P}\left(\left|X_{1}(x)-X_{1}(y)\right|>\delta, \sup _{t \leqslant 1}\left(\left|X_{t}(x)\right| \vee\left|X_{t}(y)\right|\right)>R\right) \\
& +\mathbb{P}\left(\left|X_{1}(x)-X_{1}(y)\right|>\delta, \sup _{t \leqslant 1}\left(\left|X_{t}(x)\right| \vee\left|X_{t}(y)\right|\right) \leqslant R\right), \\
& \mathbb{P}\left(\left|X_{1}(x)-X_{1}(y)\right|>\delta, \sup _{t \leqslant 1}\left(\left|X_{t}(x)\right| \vee\left|X_{t}(y)\right|\right) \leqslant R\right) \\
= & \mathbb{P}\left(\left|X_{1}^{R}(x)-X_{1}^{R}(y)\right|>\delta, \sup _{t \leqslant 1}\left(\left|X_{t}(x)\right| \vee\left|X_{t}(y)\right|\right) \leqslant R\right)
\end{aligned}
$$

and

$$
\begin{array}{r}
\mathbb{P}\left(\left|X_{1}(x)-X_{1}(y)\right|>\delta, \sup _{t \leqslant 1}\left(\left|X_{t}(x)\right| \vee\left|X_{t}(y)\right|\right)>R\right) \\
\quad \leqslant \mathbb{P}\left(\sup _{t \leqslant 1}\left(\left|X_{t}(x)\right| \vee\left|X_{t}(y)\right|\right)>R\right) \leqslant \varepsilon
\end{array}
$$


due to (3.11). Note that in order to get (3.14) we use the fact that if the process $\left(X_{t}(x)\right)_{0 \leqslant t \leqslant 1}$ stays in the ball of radius $R$ for all $t \in[0,1]$, then $\left(X_{t}^{R}(x)\right)_{0 \leqslant t \leqslant 1}$ and $\left(X_{t}(x)\right)_{0 \leqslant t \leqslant 1}$ are solutions to the same SDE [3.12), for which the solution is unique, as we explained above. Hence we obtain

$$
\begin{aligned}
\mathbb{P}\left(\left|X_{1}(x)-X_{1}(y)\right|>\delta\right) & \leqslant \varepsilon+\mathbb{P}\left(\left|X_{1}^{R}(x)-X_{1}^{R}(y)\right|>\delta\right) \\
& \leqslant \varepsilon+\frac{1}{\delta^{2}} \mathbb{E}\left|X_{1}^{R}(x)-X_{1}^{R}(y)\right|^{2},
\end{aligned}
$$

where the second step is just the Chebyshev inequality. Now from the Itô formula used similarly to 3.5 ) (cf. also the proof of Theorem 1.4 , although here we need a different local martingale than in the case where we estimate a supremum) we get

$$
\begin{aligned}
\mid X_{1}^{R}(x)- & \left.X_{1}^{R}(y)\right|^{2}=|x-y|^{2} \\
& +2 \int_{0}^{1}\left\langle X_{s}^{R}(x)-X_{s}^{R}(y), b_{R}\left(X_{s}^{R}(x)\right)-b_{R}\left(X_{s}^{R}(y)\right)\right\rangle d s \\
& +2 \int_{0}^{1}\left\langle X_{s}^{R}(x)-X_{s}^{R}(y),\left(\sigma_{R}\left(X_{s}^{R}(x)\right)-\sigma_{R}\left(X_{s}^{R}(y)\right)\right) d W_{s}\right\rangle \\
& +\int_{0}^{1}\left\|\sigma_{R}\left(X_{s}^{R}(x)\right)-\sigma_{R}\left(X_{s}^{R}(y)\right)\right\|_{\mathrm{HS}}^{2} d s \\
& +2 \int_{0}^{1} \int_{U}\left\{\left\langle X_{s}^{R}(x)-X_{s}^{R}(y), g_{R}\left(X_{s-}^{R}(x), u\right)-g_{R}\left(X_{s-}^{R}(y), u\right)\right\rangle\right. \\
& \left.+\left|g_{R}\left(X_{s-}^{R}(x), u\right)-g_{R}\left(X_{s-}^{R}(y), u\right)\right|^{2}\right\} \widetilde{N}(d s, d u) \\
& +\int_{0}^{1} \int_{U}\left|g_{R}\left(X_{s-}^{R}(x), u\right)-g_{R}\left(X_{s-}^{R}(y), u\right)\right|^{2} \nu(d u) d s \\
\leqslant & 2 C(R) \int_{0}^{1}\left|X_{s}^{R}(x)-X_{s}^{R}(y)\right|^{2} d s+M_{t},
\end{aligned}
$$

where we have used the condition 1.10$)$ for $b_{R}, \sigma_{R}$ and $g_{R}$. Moreover,

$$
\begin{aligned}
M_{t}:= & 2 \int_{0}^{1} \int_{U}\left\{\left\langle X_{s}^{R}(x)-X_{s}^{R}(y), g_{R}\left(X_{s-}^{R}(x), u\right)-g_{R}\left(X_{s-}^{R}(y), u\right)\right\rangle\right. \\
& \left.+\left|g_{R}\left(X_{s-}^{R}(x), u\right)-g_{R}\left(X_{s-}^{R}(y), u\right)\right|^{2}\right\} \widetilde{N}(d s, d u) \\
& +2 \int_{0}^{1}\left\langle X_{s}^{R}(x)-X_{s}^{R}(y),\left(\sigma_{R}\left(X_{s}^{R}(x)\right)-\sigma_{R}\left(X_{s}^{R}(y)\right)\right) d W_{s}\right\rangle
\end{aligned}
$$

is a local martingale. Thus by a localization argument and the Gronwall inequality we get

$$
\mathbb{E}\left|X_{1}^{R}(x)-X_{1}^{R}(y)\right|^{2} \leqslant A|x-y|^{2} e^{B t}
$$


for some constants $A, B>0$, and thus

$$
\mathbb{P}\left(\left|X_{1}(x)-X_{1}(y)\right|>\delta\right) \leqslant \varepsilon+\frac{A}{\delta^{2}}|x-y|^{2} e^{B t} .
$$

Once we have (3.16), we proceed exactly as in [1, proof of Proposition 4.3]. Namely, we can show that for any sequence $x_{n} \rightarrow x$ in $\mathbb{R}^{d}$ we have $X_{1}\left(x_{n}\right) \rightarrow$ $X_{1}(x)$ in probability. From this we infer that for any $f \in \mathcal{C}_{b}\left(\mathbb{R}^{d}\right)$ we have $p_{1} f\left(x_{n}\right) \rightarrow p_{1} f(x)$, from which we get the Feller property of $\left(p_{t}\right)_{t \geqslant 0}$. The details of this last step can be found [1, p. 321].

Proof of Theorem 2.1. Note that (2.3) obviously implies (1.3) and hence under the assumptions of Theorem 2.1, the assumptions of Lemma 2.2 are satisfied. Thus the semigroup $\left(p_{t}\right)_{t \geqslant 0}$ associated with the solution $\left(X_{t}\right)_{t \geqslant 0}$ of $1.1 p$ is Feller. Hence, if we can show (2.2), then we can just use the Krylov-Bogolyubov method presented at the beginning of Section 2 and conclude the proof.

In order to prove $(2.2)$, we apply the Itô formula to $\left|X_{t}(x)\right|^{2}$ as in $(3.5)$ and then proceed as in the proof of Lemma 2.2 above, where we apply the Itô formula to obtain 3.15). However, unlike in 3.15), here we need to obtain the term $e^{B t}$ with a negative constant $B$ in order to guarantee (2.1), the boundedness in probability at infinity. Thus we need to use the differential version of the Gronwall inequality and not the integral one (cf. [17, Remark 2.3]). This is however not a problem, since by using (2.3) and choosing a local martingale accordingly, we can obtain

$$
\mathbb{E}\left|X_{t}(x)\right|^{2} \leqslant \mathbb{E}\left|X_{s}(x)\right|^{2}-K \int_{s}^{t}\left(\mathbb{E}\left|X_{r}(x)\right|^{2}-\frac{M}{K}\right) d r
$$

for any $0 \leqslant s \leqslant t$. Thus by the differential version of the Gronwall inequality we have $\mathbb{E}\left|X_{t}(x)\right|^{2}-M / K \leqslant|x|^{2} e^{-K t}$, which gives 2.2 and finishes the proof.

Proof of Theorem 1.1. Under our assumptions we can prove that the coefficients of (1.1) are locally bounded in the sense of $(1.8)$ (cf. the discussion at the beginning of Section 3). Then combining Lemma 1.1 and Theorem 1.4, we see that for every $R \geqslant 1$ there exists a unique strong solution $\left(X_{t}^{R}\right)_{t \geqslant 0}$ to 1.1 with the coefficients replaced by $b_{R}, \sigma_{R}$ and $g_{R}$ from Lemma 1.1. If we consider a sequence $\left\{\left(X_{t}^{n}\right)_{t \geqslant 0}\right\}_{n \in \mathbb{N}}$ of such solutions and define stopping times $\left\{\tau_{n}\right\}_{n \in \mathbb{N}}$ as in (3.13, then we can show that for $n \leqslant m$ we have $\tau_{n} \leqslant \tau_{m}$, and consequently $\tau:=\lim _{n \rightarrow \infty} \tau_{n}$ and

$$
X_{t}:=\lim _{n \rightarrow \infty} X_{t}^{n} \quad \text { almost surely on }[0, \tau]
$$

are well defined. We just need to show that $\left(X_{t}\right)_{t \geqslant 0}$ is indeed a solution of 1.1 . 
By the construction of the coefficients in Lemma 1.1 we see that

$$
\begin{aligned}
X_{t \wedge \tau_{n}}=X_{t \wedge \tau_{n}}^{n}= & X_{0}+\int_{0}^{t \wedge \tau_{n}} b\left(X_{s}\right) d s+\int_{0}^{t \wedge \tau_{n}} \sigma\left(X_{s}\right) d W_{s} \\
& +\int_{0}^{t \wedge \tau_{n}} \int_{U} g\left(X_{s-}, u\right) \tilde{N}(d s, d u) .
\end{aligned}
$$

Therefore it remains to show that $\tau=\infty$ a.s., which can be done exactly as in [1, Theorem 3.1]. Namely, using the global one-sided linear growth condition (1.3) we can show that $\mathbb{E}\left|X_{t \wedge \tau_{n}}\right|^{2} \leqslant\left(\mathbb{E}\left|X_{0}\right|^{2}+K t\right) e^{K t}$ for some constant $K>0$ and then, after proving $n^{2} \mathbb{P}\left(\tau_{n}<t\right) \leqslant \mathbb{E}\left|X_{t \wedge \tau_{n}}\right|^{2}$, we see that $\tau_{n} \rightarrow \infty$ in probability, and thus, for a subsequence, almost surely.

Acknowledgments. I would like to thank Zdzisław Brzeźniak for discussions regarding the contents of [1]. I am also grateful to the anonymous referees for their useful remarks and suggestions.

This work was financially supported by the Bonn International Graduate School of Mathematics.

\section{REFERENCES}

[1] S. Albeverio, Z. Brzeźniak and J.-L. Wu, Existence of global solutions and invariant measures for stochastic differential equations driven by Poisson type noise with non-Lipschitz coeffcients, J. Math. Anal. Appl. 371 (2010), 309-322.

[2] D. Applebaum, Lévy Processes and Stochastic Calculus, 2nd ed., Cambridge Univ. Press, 2009.

[3] A. Arapostathis, A. Biswas and L. Caffarelli, On a class of stochastic differential equations with jumps and its properties, arXiv:1401.6198v4 (2015).

[4] K. Bichteler, J.-B. Gravereaux and J. Jacod, Malliavin Calculus for Processes with Jumps, Stochastics Monographs, 2. Gordon and Breach, New York, 1987.

[5] Z. Brzeźniak, W. Liu and J. Zhu, Strong solutions for SPDE with locally monotone coefficients driven by Lévy noise, Nonlinear Anal. Real World Appl. 17 (2014), 283-310.

[6] G. Da Prato, D. Gątarek and J. Zabczyk, Invariant measures for semilinear stochastic equations, Stochastic Anal. Appl. 10 (1992), 387-408.

[7] I. Gyöngy and N. V. Krylov, On stochastic equations with respect to semimartingales. I, Stochastics 4 (1980/81), 1-21.

[8] R. Z. Has'minskii, Stochastic Stability of Differential Equations, Sijthoff \& Noordhoff, Alphen aan den Rijn, 1980.

[9] N. Ikeda and S. Watanabe, Stochastic Differential Equations and Diffusion Processes, 2nd ed., North-Holland and Kodansha, 1989.

[10] T. Komorowski and A. Walczuk, Central limit theorem for Markov processes with spectral gap in the Wasserstein metric, Stochastic Process. Appl. 122 (2012), 2155-2184.

[11] N. V. Krylov and B. L. Rozovskii, Stochastic evolution equations, J. Soviet Math. 16 (1981), $1233-1277$.

[12] W. Liu and J. M. Tölle, Existence and uniqueness of invariant measures for stochastic evolution equations with weakly dissipative drifts, Electron. Comm. Probab. 16 (2011), 447-457.

[13] Y. Ma, Transportation inequalities for stochastic differential equations with jumps, Stochastic Process. Appl. 120 (2010), 2-21. 
[14] M. B. Majka, Transportation inequalities for non-globally dissipative SDEs with jumps via Malliavin calculus and coupling, Ann. Inst. Henri Poincaré Probab. Statist. 55 (2019), 2019_ 2057.

[15] J.-L. Menaldi and M. Robin, Invariant measure for diffusions with jumps, Appl. Math. Optim. 40 (1999), 105-140.

[16] C. Prévôt and M. Röckner, A Concise Course on Stochastic Partial Differential Equations, Springer, Berlin, 2007.

[17] J. Shao and C. Yuan, Transportation-cost inequalities for diffusions with jumps and its application to regime-switching processes, J. Math. Anal. Appl. 425 (2015), 632-654.

[18] R. Situ, Theory of Stochastic Differential Equations with Jumps and Applications, Springer, New York, 2005.

[19] J. Wang, $L^{p}$-Wasserstein distance for stochastic differential equations driven by Lévy processes, Bernoulli 22 (2016), 1598-1616.

[20] L. Wu, Transportation inequalities for stochastic differential equations of pure jumps, Ann. Inst. Henri Poincaré Probab. Statist. 46 (2010), 465-479.

\author{
Mateusz B. Majka \\ Institute for Applied Mathematics \\ University of Bonn \\ Endenicher Allee 60 \\ 53115 Bonn, Germany \\ E-mail:majka@uni-bonn.de
}


